\title{
Caracterización morfométrica de la cuenca del Salado Bajo, Región de Atacama, Chile
}

\author{
Morphometric characterization of the Salado Bajo basin, Atacama Region, Chile \\ Historial del artículo \\ Recibido: \\ 10 de agosto de 2021 \\ Revisado \\ 10 de noviembre de 2021 \\ Aceptado: \\ 18 de noviembre de 2021 \\ Keyla Manuela Alencar da Silva Alves ${ }^{\mathrm{a}}$, María Carolina Parodi Dávila ${ }^{\mathrm{b}}$, \\ Erik Daniel Zimmermann García ${ }^{\mathrm{c}}$, Daniel Rodrigues de Lira ${ }^{\mathrm{d}}$, \\ Kleython de Araújo Monteiro ${ }^{\mathrm{e}}$ \\ a Departamento de Industria, Facultad de Ingeniería, Universidad Tecnológica Metropolitana. Correo electrónico: keyla.dasilva@ \\ utem.cl. http://orcid.org/0000-0001-7635-2430 \\ ${ }^{b}$ Departamento de Industria, Facultad de Ingeniería, Universidad Tecnológica Metropolitana. http://orcid.org/0000-0002-2132-5168 \\ ' Departamento de Hidráulica, Facultad de Ciencias Exactas, Ingeniería y Agrimensura, Universidad Nacional de Rosario. \\ https://orcid.org/0000-0003-4424-7737 \\ ${ }^{d}$ Departamento de Geografía, Universidade Federal de Pernambuco. http://orcid.org/0000-0001-9559-2480 \\ e Departamento de Geografía, Campus A. C. Simões, Universidade Federal de Alagoas, Maceió. http://orcid.org/0000-0003- \\ 4829-3722
}

Palabras clave

Atacama, cuenca hidrográfica, geomorfología, morfometría

\section{Keywords}

Atacama, geomorphology, morphometry, watershed

\section{Resumen}

El análisis en conjunto de parámetros morfométricos puede ser usado en la evaluación de la susceptibilidad del terreno, y se presenta como una técnica de levantamiento topográfico simple y de bajo costo para la zonificación ambiental y la gestión de desastres. Este estudio presenta el análisis de un conjunto de índices morfométricos aplicados a la cuenca hidrográfica del Salado Bajo-Región de Atacama (Chile), con el objetivo de determinar las características geomorfológicas que condicionan el escenario previo al evento meteorológico gatillante de los flujos de detritos en cuencas de zonas áridas. La metodología se basó en la caracterización física por medio del análisis de: parámetros morfométricos, análisis hipsométrico, jerarquización de los canales y densidad de drenaje. Los principales resultados obtenidos demostraron que la forma alargada de la cuenca es un factor condicionante a la ocurrencia de flujos de detritos. Además, los resultados de pendiente media de la cuenca y la velocidad media de flujo indican que el terreno de la cuenca es fuertemente accidentado y que la velocidad de escorrentía y el poder de arrastre del material erosionada son altos.

\begin{abstract}
The analysis of various morphometric parameters can be used to evaluate the susceptibility of the surface, but it can also be applied as a simple and inexpensive topographic survey technique for environmental zoning and disaster management. This study presents an analysis of a set of morphometric indices applied to the hydrographic basin of the Salado Bajo-Atacama Region (Chile), with the objective of determining the geomorphological characteristics that condition the previous scenario of a meteorological event triggering the debris flows in hydrographic basins of arid zones. The applied methodology is based on physical characterization through the analysis of morphometric parameters, hypsometric analysis, channel hierarchy and drainage density. The main results obtained demonstrate that the elongated shape of the basin is a conditioning factor for occurrences of debris flows. Also, the results of the average slope of the basin and the average flow velocity indicate that the terrain of the basin is heavily hilly and that the flow rate and the dragging power of the eroded material are high.
\end{abstract}




\section{Introducción}

El contexto físico-geográfico de una cuenca hidrográfica representa el marco espacial para la caracterización de diversos sistemas ambientales, así como también es el marco referencial para la planificación territorial de los recursos naturales (Shivhare et al., 2021). Una cuenca hidrográfica posee funcionamientos múltiples, como colector de lluvias en el paisaje, receptación de precipitaciones y conduce el escurrimiento superficial y subsuperficial. Sin embargo, estas funciones dependen de las condiciones climáticas y de las características físicas de la cuenca. La interacción entre las variables climáticas y las variables físicas del paisaje resultan en una serie de procesos de orígenes hidrológicos y geomorfológicos, donde comprender la dinámica de tales procesos naturales empieza en la caracterización de los parámetros morfométricos de la cuenca (Cordeiro, et al., 2019).

En un contexto geomorfológico general, el análisis morfométrico proporciona descripciones cuantitativas de geometría y topología. Las características físicas del paisaje que facilitan la determinación del comportamiento, escala, complejidad y variabilidad de estructuras geológicas, por medio de índices numéricos que pueden correlacionarse con parámetros físicos. De esta forma, el arquetipo del análisis morfométrico en geomorfología es la morfometría de los sistemas fluviales (Godínez-Tamay et al., 2020; Oliveira, et al., 2019).

Los parámetros morfométricos son un conjunto de ecuaciones que describen los elementos geomórficos típicos para la caracterización física de una cuenca hidrográfica. El análisis de la morfométrica de cuencas es un método cuantitativo sistémico que corrobora con la descripción evolutiva y dinámica de la cuenca. El empleo de los parámetros morfométricos en los estudios previos de caracterización hidrológica y geomorfológica son fundamentales e indispensables, principalmente cuando estos estudios de base van integrados a estudios avanzados de la dinámica hidro geomorfológica (Soares et al., 2016). Esta prioridad relacionada a la morfometría fluvial está vinculada a cómo los resultados de los parámetros morfométricos contribuyen sustancialmente con la interpretación del comportamiento morfodinámico e hidrológico, y de cómo estos resultados contribuyen con la validación de modelaciones y simulaciones de eventos hidro geomorfológicos tales como inundaciones y flujos (Fonseca \& Augustin, 2014).
La morfometría de la cuenca, desde el análisis de la red de drenaje, jerarquía de los canales y de los diferentes índices que definen la forma y las características físicas de ella (rugosidad, ancho, longitud, compacidad, elevación, pendiente, etc.), llevan a comprender la morfodinámica de diversos procesos geomorfológicos, en los cuales los flujos de detritos constituyen un proceso morfogenético activo alterando el relieve (Abuzied et al., 2016; Buccolini, et al., 2012; Gil et al., 2019; Jothimani et al., 2021; Othman et al., 2018 ). De acuerdo a los valores de cada parámetro e índice, o de las combinaciones de ellos, conjuntamente con parámetros climáticos, hidrológicos y sedimentológicos es posible predecir genéricamente la susceptibilidad de algunas cuencas a eventos tales como inundaciones, crecidas, flujos, remociones en masa, etc. (Pereira \& Mendes, 2018).

La cuenca hidrográfica del Salado Bajo, ubicada en la Región de Atacama (Chile), fue seleccionada para este estudio por poseer registros históricos de diversos eventos de flujos de detritos (Contreras et al., 2015; Cortés et al., 2015; Da Silva Alves et al., 2020; Ramirez \& Perez, 2011; Monroy, 2017; Villegas, 2017). Para validar la metodología empleada se optó por elegir una cuenca con antecedentes de ocurrencia de tales eventos, con el fin de caracterizar los factores condicionantes. Estos factores se refieren a las características hidrológicas y geomorfológicas que condicionan el escenario previo al evento meteorológico gatillante de los flujos de detritos en cuencas de zonas áridas.

Ante la necesidad de caracterizar los factores condicionantes, este trabajo tiene como objetivo calcular una serie de índices y parámetros morfométricos, hipsométricos e hídricos que definan los aspectos hidrológicos y geomorfológicos de la cuenca del río Salado Bajo.

\section{Materiales y métodos}

\section{Área de estudio: antecedentes geológicos y geomorfológicos}

La cuenca hidrográfica del Salado Bajo está ubicada en la Región de Atacama, Chile. La parte más alta de la cuenca se posiciona en los 1.995 m.s.n.m. y el canal principal posee longitud de aproximadamente $78,71 \mathrm{~km}$, lo que se encuentra enlazado con el orden de sus afluentes, los cuales se unen en una sola desembocadura en la comuna de Chañaral. 


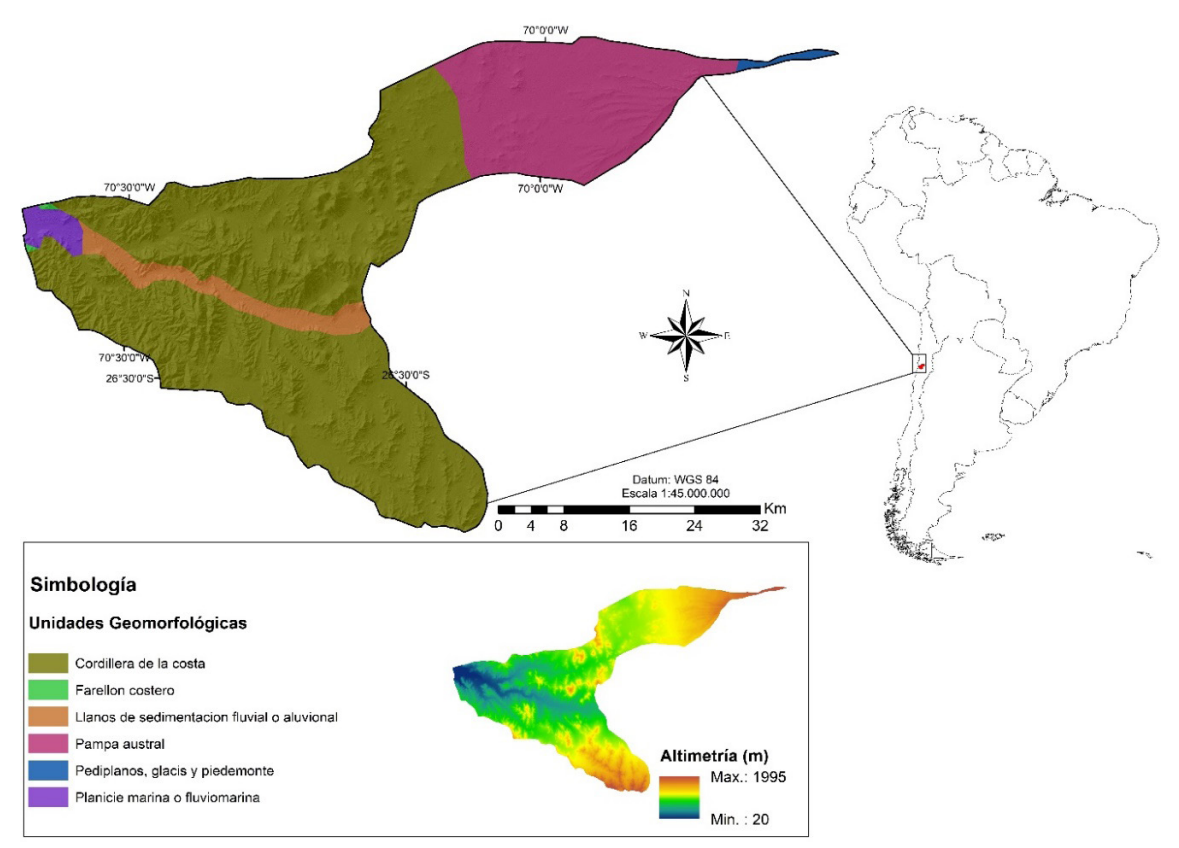

Figura 1. Localización del área de estudio, unidades geomorfológicas (elaborado a partir de la base de datos georreferenciados de la Universidad de la Frontera) y mapa de elevación. Fuente: elaboración propia

Las unidades geomorfológicas que componen la cuenca son la planicie fluviomarina con cordillera de la Costa, y partes de la pampa ondulada y de la cordillera Domeyko (Lorca, 2016). La altitud aumenta desde las planicies fluviomarinas hacia la cordillera Domeyko, aumentando gradualmente hasta la pampa ondulada. La cordillera de la Costa no suele superar los $1.500 \mathrm{~m}$, y la cordillera Domeyko alcanza los $3.000 \mathrm{~m}$, donde los cauces de los ríos se orientan en dirección norte-sur, principalmente por influencia de las fallas (figura 1). Los suelos predominantes son los yermosoles cálcicos y los cambisoles ócricos, respectivamente ${ }^{1}$.

La geología incorpora gran cantidad de depósitos no consolidados donde se ubican las quebradas, así como formaciones rocosas de edades Paleozoicas y Cenozoicas, tal como se puede observar en la figura 2. La litología, en general, está dividida entre rocas intrusivas y estratificadas (sedimentarias y volcanosedimentarias) (Cornejo et al., 1999; Godoy \& Lara, 2005).

A lo largo de la cuenca, las fallas geológicas cruzan los canales del río Salado, quebrada Saladillo y quebrada las Ánimas, modelando los aspectos morfológicos de los valles (Grijalba, 2016). El sistema de fallas de Atacama se destaca en trazas norte sur y fallas secundarias de sentido noreste (falla Cerro Salado) entre las comunas de Chañaral y Diego de Almagro, donde se observa un manteo hacia el este de las formaciones del Jurásico (formación La Negra) y del Cretácico (formación llanta) (Godoy \& Lara, 1998).

Entre las formaciones del Cuaternario se destacan los depósitos eólicos y de playa de arenas finas; los depósitos coluviales de composición monomíctica ubicado en las laderas de los cerros; los depósitos aluviales compuestos por gravas, arenas y limos de granulometría variada asociados a los flujos de detritos (Tomlinson et al., 1999). Se incluyen también los depósitos antrópicos (Holoceno) asociados a depósitos de relave del yacimiento El Salvador (Cornejo et al., 1998).

\section{Parámetros morfométricos}

El procedimiento técnico propuesto para este estudio se basó en caracterizar físicamente la cuenca del Salado Bajo por medio del análisis de los parámetros morfométricos, análisis hipsométrico, jerarquización de los canales y densidad de drenaje. Los parámetros morfométricos fueron seleccionados de acuerdo con la relación que se establece con la hidrología y el relieve de la cuenca (tabla 1).

${ }^{1}$ Base de datos geológicos de Servicio Nacional de Geología y Minería. 


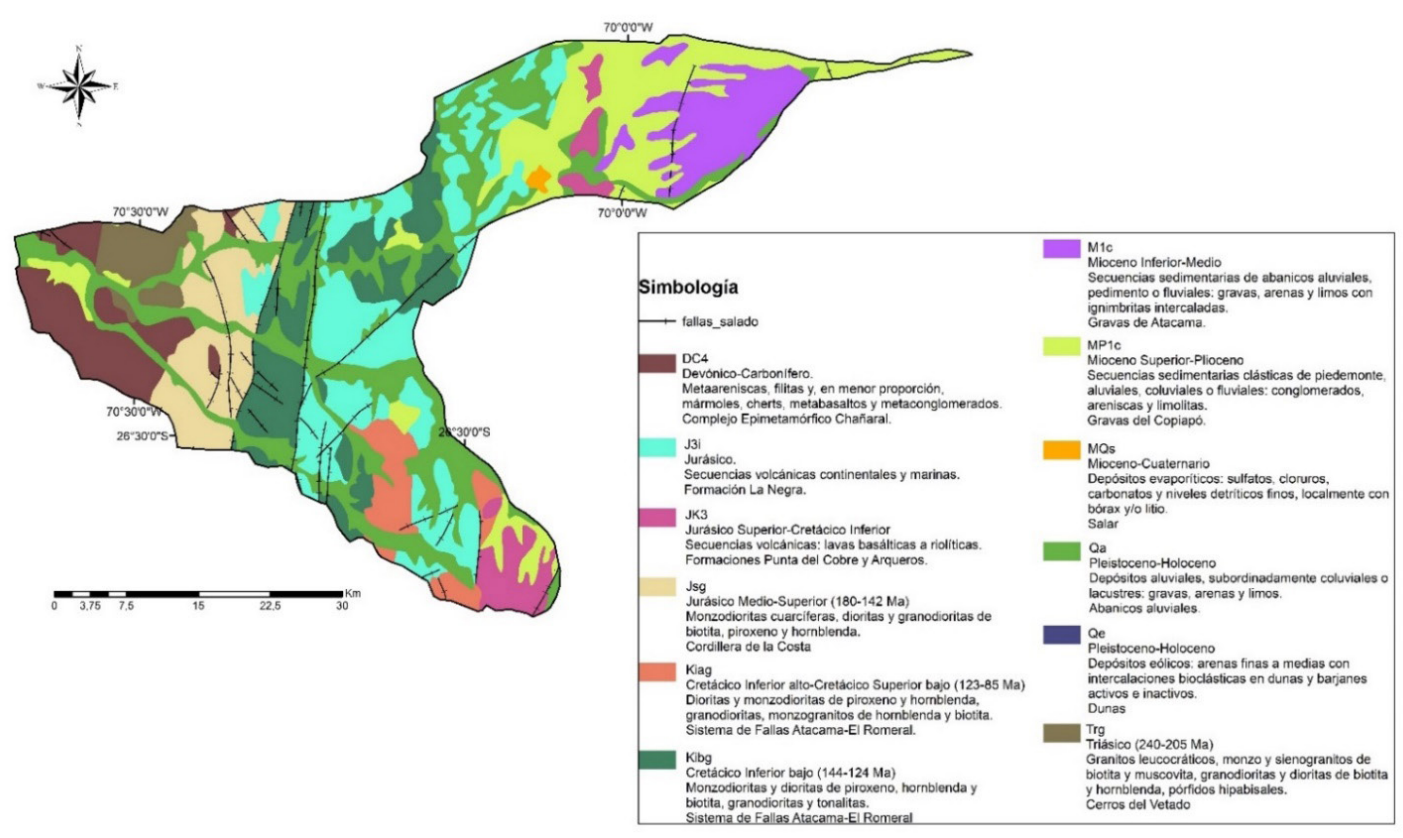

Figura 2. Mapa geológico de la cuenca del Salado bajo (elaborado a partir de la base de datos de SERNAGEOMIN). Fuente: elaboración propia

Tabla 1

Parámetros morfométricos seleccionados y utilizados para la caracterización de la cuenca del Salado Bajo.

\begin{tabular}{|c|c|c|}
\hline Variable & Parámetro (unidades; sigla) & Expresión o herramienta de cálculo \\
\hline \multirow{10}{*}{ 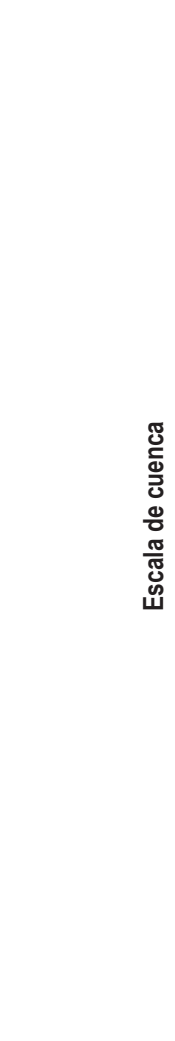 } & Área $(\mathrm{km} 2 ; \mathrm{A})$ & SIG \\
\hline & & $L=\frac{P}{4}+\sqrt{\frac{P^{2}}{16}}-A$ \\
\hline & Rectángulo equivalente (km; L) & $l=\frac{P}{4}-\sqrt{\frac{P^{2}}{16}-A}$ \\
\hline & & $\begin{array}{l}\mathrm{L}=\text { lado mayor del rectángulo en } \mathrm{km} \text {. } \\
\mathrm{l=} \text { lado menor del rectángulo en } \mathrm{km} \text {. } \\
\mathrm{P}=\text { perímetro en } \mathrm{km} \text {. } \\
\mathrm{A}=\text { área de la cuenca en } \mathrm{km}^{2} \text {. }\end{array}$ \\
\hline & Área de la vertiente mayor (este) (km2) & SIG \\
\hline & Área de la vertiente menor (oeste) (km2) & SIG \\
\hline & Perímetro (km) & SIG \\
\hline & Longitud $(\mathrm{km})$ & SIG \\
\hline & Ancho máximo $(\mathrm{km})$ & SIG \\
\hline & Diámetro (km) & $\begin{array}{l}D=\sqrt{ } 4^{*} A / N \\
D=\text { Diámetro. } \\
A=\text { área de la cuenca en } \mathrm{km} 2 . \\
N=\text { número constante equivalente a } 3,1415 .\end{array}$ \\
\hline
\end{tabular}




\begin{tabular}{|c|c|c|}
\hline & Altitud mínima (msnm) & SIG \\
\hline & Altitud máxima (msnm) & SIG \\
\hline & Altitud media (msnm) & SIG \\
\hline & Pendiente media ( $\mathrm{m} / \mathrm{m}$ en porcentaje) & SIG \\
\hline & Elongación (adimensional) & $\begin{array}{l}\mathrm{E}=\mathrm{A} / \mathrm{L} \\
\mathrm{E}=\text { elongación. } \\
\mathrm{A}=\text { área de la cuenca en } \mathrm{km}^{2} \text {. } \\
\mathrm{L}=\text { longitud de la cuenca en } \mathrm{km} \text {. }\end{array}$ \\
\hline & Perímetro relativo de crenulación (adimensional) & $\begin{array}{l}\text { Prc }=\text { P2/A } \\
\text { Prc= Perímetro relativo de crenulación. } \\
\mathrm{P} 2=\text { perímetro al cuadrado en } \mathrm{km} . \\
\mathrm{A}=\text { área de la cuenca en } \mathrm{km}^{2} .\end{array}$ \\
\hline & $\begin{array}{l}\text { Coeficiente de compacidad (Gravelius) } \\
\text { (adimensional) }\end{array}$ & $\begin{array}{l}\mathrm{Kc}=0.28 \mathrm{P} / \sqrt{ } \mathrm{A} \\
\mathrm{K} \mathrm{c}=\text { Coeficiente de compacidad (Gravelius). } \\
\mathrm{P}=\text { perímetro en } \mathrm{km} . \\
\mathrm{A}=\text { área de la cuenca en } \mathrm{km}^{2} .\end{array}$ \\
\hline & Índice de alargamiento (adimensional) & $\begin{array}{l}\mathrm{La}=\mathrm{L} / \mathrm{a}_{\max } \\
\mathrm{La}=\text { índice de alargamiento (adimensional). } \\
\mathrm{L}=\text { longitud máxima de la cuenca en } \mathrm{km} . \\
\text { amax = ancho máximo de la cuenca en } \mathrm{km} \text {. }\end{array}$ \\
\hline & Índice de homogeneidad (adimensional) & $\begin{array}{l}\text { Ih=a/(LAx) } \\
\text { Ih= índice de homogeneidad. } \\
a=\text { ancho máximo en } \mathrm{km} . \\
\text { Lax= longitud axial. }\end{array}$ \\
\hline $\begin{array}{l}\frac{\pi}{0} \\
\frac{\pi}{0} \\
\frac{1}{0}\end{array}$ & Índice de asimetría (adimensional) & $\begin{array}{l}\text { Is }=100(\mathrm{Ar} / \mathrm{At}) \\
\mathrm{Is}=\text { índice de asimetría. } \\
\mathrm{Ar}=\text { área de la vertiente mayor en } \mathrm{km}^{2} . \\
\mathrm{At}=\text { área de la vertiente menor en } \mathrm{km}^{2} .\end{array}$ \\
\hline 8 & Factor forma (adimensional) & $\begin{array}{l}\mathrm{Ff}=\mathrm{A} / \mathrm{L}^{2} \\
\mathrm{Ff}=\text { factor de forma. } \\
\mathrm{A}=\text { área de la cuenca en } \mathrm{km} 2 . \\
\mathrm{L} 2=\text { cuadrado de la longitud máxima del cauce en } \mathrm{km} \text {. }\end{array}$ \\
\hline 离 & Alargamiento de Caquot (adimensional) & $\begin{array}{l}\mathrm{Ca}=\mathrm{E} / \sqrt{ } \mathrm{S} \\
\mathrm{Ca}=\text { alargamiento de Caquot. } \\
\mathrm{E}=\text { recorrido más largo entre la periferia y la salida de la cuenca } \\
\text { en } \mathrm{km} . \\
\sqrt{ } \mathrm{S}=\text { raíz cuadrada de la superficie de la longitud del lado de un } \\
\text { cuadrado de área equivalente. }\end{array}$ \\
\hline & Radio de elongación (adimensional) & $\begin{array}{l}\mathrm{Re}=\mathrm{D} / \mathrm{L} \\
\mathrm{Re}=\text { radio de elongación. } \\
\mathrm{D}=\text { diámetro de un círculo de la misma área de la cuenca. } \\
\mathrm{L}=\text { longitud de la cuenca. }\end{array}$ \\
\hline & Radio de circularidad (adimensional) & $\begin{array}{l}\mathrm{Rc}=4 \pi \mathrm{A} / \mathrm{P}^{2} \\
\mathrm{Rc}=\text { radio de circularidad. } \\
\mathrm{A}=\text { área de la cuenca en } \mathrm{km}^{2} . \\
\mathrm{P} 2=\text { área de un círculo de igual perímetro en } \mathrm{km} .\end{array}$ \\
\hline & Índice de forma (adimensional) & $\begin{array}{l}\text { If }=P / 2\left(\pi^{*} A\right)^{1 / 2} \\
\text { If = índice de forma. } \\
P=\text { perímetro de la cuenca en } \mathrm{km} . \\
A=\text { área de la cuenca en } \mathrm{km}^{2} .\end{array}$ \\
\hline & $\begin{array}{l}\text { Índice entre el largo y el área de la cuenca } \\
\text { (adimensional) }\end{array}$ & $\begin{array}{l}\text { IcO= D/A } \\
\text { IcO= índice entre el largo y el área de la cuenca. } \\
D=\text { diámetro de un círculo de la misma área de la cuenca. } \\
A=\text { área de la cuenca en } \mathrm{km}^{2} \text {. }\end{array}$ \\
\hline & Coeficiente de forma (adimensional) & $\begin{array}{l}\mathrm{Cf}=\mathrm{a}_{\mathrm{m}} / \mathrm{L} \\
\mathrm{Cf}=\text { coeficiente de forma. } \\
\mathrm{a}_{\text {max }}=\text { ancho máximo de la cuenca en } \mathrm{km} . \\
\mathrm{L}=\text { longitud máxima de la cuenca en } \mathrm{km} .\end{array}$ \\
\hline & Coeficiente de redondez (adimensional) & $\begin{array}{l}\mathrm{Cr}=\pi \mathrm{L}^{2} / 4 \mathrm{~A} \\
\mathrm{Cr}=\text { coeficiente de redondez. } \\
\mathrm{L}=\text { longitud máxima de la cuenca en } \mathrm{km} \text {. } \\
\mathrm{A}=\text { área de la cuenca en } \mathrm{km}^{2} \text {. }\end{array}$ \\
\hline
\end{tabular}

Fuente: Da Conceição Dornellas et al. (2020), De Antueno et al. (2020) y Bogale (2021). 


\section{Curva hipsométrica}

La curva hipsométrica (Harlin, 1978) permitió caracterizar la distribución de masa en la cuenca desde la cabecera hasta la desembocadura. Se obtuvo a partir de los valores correspondientes a las diferentes alturas de la cuenca referidos a la máxima y las abscisas. Las curvas que presentan valores de área superiores a las alturas correspondientes, referidos al área total de la cuenca, normalmente posee forma sigmoidal, cóncava hacia arriba en la parte superior y convexa en la parte inferior. El grado de sinuosidad es muy variable, lo mismo que la pendiente en el punto de inflexión. Cuando las curvas hipsométricas presentan variaciones, ya sea por no obedecer al padrón teórico referencial o por presentar más de un punto de inflexión, el resultado puede relacionarse con controles tectónicos o litológicos. El cálculo de curva se da por la siguiente ecuación 1:

$$
f(x)=a_{0}+a_{1} x+a_{2} x^{2}+a_{3} x^{3}
$$

La regresión de la curva hipsométrica permite la extracción de los coeficientes $a_{x}$, aplicados en la integral de área (A) de la curva, demostrada en la ecuación 2:

$$
A=\int_{0}^{1} a_{0}+a_{1} x+a_{2} x^{2}+a_{3} x^{3} f(x) d x
$$

$f(x)$ representa el eje $\mathrm{x}$ y es el centróide de la función $H i$, en la ecuación 3 :

$$
H i=\frac{1}{A} x f(x) d x
$$

El valor del área relativa (integral hipsométrica) demuestra el estado de desarrollo de la cuenca; valores superiores al $60 \%$ indican desequilibrio en el funcionamiento dinámico de la cuenca en su etapa de juventud, valores entre el $40-45 \%$, representan equilibrio en su etapa de madurez y valores inferiores a $30 \%$ implican etapa de senectud (Harsha et al., 2020).

\section{Jerarquización de los canales}

Se usó el método de clasificación de Strahler (1957, 1989). Este considera que distintos tipos de canales se ramifican, y que de acuerdo con el tipo de ramificación se puede determinar el orden del canal y así jerarquizar toda una red de drenaje. Los puntos de intersección entre dos segmentos de un canal se clasificaron como nodos internos. Los puntos que daban origen a un segmento de canal (Bertolini et al., 2019) se clasificaron como nodos externos. Según este método los canales se ordenaron de acuerdo los siguientes criterios:
Cuando dos tramos de canal del mismo orden, i, se unen en un nodo interior se origina un canal de orden superior, $\mathrm{i}+1$, aguas abajo; en la intersección de dos tramos de distinto orden se conserva el tramo de mayor orden; el orden de la cuenca, w, es el mismo del canal de mayor orden.

Se presentan relaciones claras entre los números y longitudes de los canales de cada orden. Estas demuestran que el número de canales de distinto orden en una cuenca en orden decreciente, según la relación logarítmica de la fórmula 4:

$\mathrm{N}_{\mathrm{s}}=\mathrm{a} \cdot \mathrm{b}^{\mathrm{s}}$

En la que $\mathrm{N}_{\mathrm{s}}=$ al número de ríos de cualquier orden y $\mathrm{s}$ $=$ orden. La longitud de los ríos de cualquier orden (Ls) disminuye con el orden u órdenes de la misma manera. $\mathrm{Ls}=\mathrm{a} \cdot \mathrm{b}^{\mathrm{s}}=$ Los factores de $\mathrm{a}$ y $\mathrm{b}, \mathrm{x}$ e y variarán según el continente o la zona climática. Donde la mayor parte de la longitud del canal de toda cuenca está situada en muchos de los afluentes pequeños de orden más bajo.

El tiempo de concentración del canal principal fue calculado en base a la fórmula de Passini:

$$
\mathrm{T}_{\mathrm{c}}=\frac{(\mathrm{SL})^{\frac{1}{3}}}{\mathrm{i}^{0.5}}, \quad 0.04 \leq \mathrm{Tc} \leq 0.13
$$

$\mathrm{Tc}=$ tiempo de concentración (horas), $\mathrm{i}=$ pendiente media del cauce principal (\%), $\mathrm{S}=$ área de la cuenca $\left(\mathrm{km}^{2}\right), \mathrm{L}=$ longitud del cauce principal $(\mathrm{km})$.

\section{Densidad de la red de drenaje}

La densidad de drenaje es la relación entre longitud total de los cursos de agua irregulares y regulares, y la superficie total de la cuenca (Bajirao, Kumar \& Kumar, 2019; Rahmati et al., 2019). Este parámetro es muy representativo respecto a la geomorfología de la cuenca, pues posee relación directa con la rugosidad, topografía y pendiente del terreno. Según los resultados que se obtenga, los valores mínimos de esta relación están asociados a superficies poco erosionables y pendientes planas, siendo esta cuenca pobremente drenada. Mientras que, los valores más altos están relacionados a suelos impermeables, elevadas tasas de erosión y pendientes pronunciadas, donde las precipitaciones intervienen rápidamente sobre las descargas de los ríos, evacuando el agua en menos tiempo (Bhattacharya et al., 2021). Este parámetro se calculó a partir de la ecuación 6 : 
$D d=\frac{L i}{A}$

En que $\mathrm{Dd}=$ Densidad de drenaje $; \mathrm{Li}=$ Longitud total de los canales en km; $\mathrm{A}=$ Superficie de la Cuenca en $\mathrm{km}^{2}$. Los valores referenciales, se muestran a continuación (tabla 2).

\section{Resultados}

Los parámetros que representan la escala de la cuenca fueron calculados con el objetivo de subsidiar valores para los cálculos de los índices de forma y gradiente. Los parámetros de escala de la cuenca presentan área de $1.856,868 \mathrm{~km}^{2}$, perímetro de $297,045 \mathrm{~km}$, diámetro $48,623 \mathrm{~km}$, área de la vertiente mayor $1.193,102 \mathrm{~km}^{2}$ y área de la vertiente menor $663,765 \mathrm{~km}^{2}$, ancho máximo $39,422 \mathrm{~km}$, longitud 77,241 km. De este conjunto de parámetros todos sirvieron como datos complementarios para los demás parámetros, excepto los resultados del rectángulo equivalente mayor y menor, respectivamente $\mathrm{L}=134,77 \mathrm{~km}$ y $1=13,75 \mathrm{~km}$. Estos resultados no presentaron ninguna significancia para la caracterización propuesta.

Los cálculos de las altitudes máxima (1.995 msnm), mínima (20 msnm) y media (1.019 msnm) indican que la altitud es asimétricamente positiva. La superficie de la cuenca con altitudes superiores a la media es menor que la superficie con altitudes inferiores al valor medio (tabla 3).

El resultado de la pendiente media $(18,59 \mathrm{~m} / \mathrm{m}$ en porcentaje) indica que la cuenca posee relieve fuertemente accidentado. Los parámetros de radio elongación $(0,629)$, elongación $(0,629)$, alargamiento de Caquot $(1,792)$, índice de alargamiento $(1,959)$, indican que la cuenca posee forma alargada.

El coeficiente de forma $(0,323)$, coeficiente de redondez $(2,523)$, factor de forma $(0,311)$ y el perímetro relativo de crenulación $(47,518)$ también definen la cuenca como alargada, y el índice de forma $(1,944)$ agrega que la cuenca además de alargada es moderadamente lobular. Otro parámetro de forma, radio de circularidad $(0,264)$, demuestra que la circularidad de la cuenca es baja, lo que reafirma los resultados anteriores que la definen como alargada.

El índice de compacidad (Kc), que también es un parámetro de forma, se define como el cociente entre el perímetro de la cuenca, respecto al perímetro de un
Tabla 2

Rangos aproximados de la Densidad de drenaje.

\begin{tabular}{lc}
\hline $\begin{array}{l}\text { Densidad de drenaje } \\
\text { (valores aproximados) }\end{array}$ & Clases \\
\hline 0,1 a 1,8 & Baja \\
\hline 1,9 a 3,6 & Moderada \\
\hline 3,7 a 5,6 & Alta \\
\hline
\end{tabular}

Fuente: Lin et al. (2021).

Tabla 3

Distribución de áreas por rangos hipsométricos.

\begin{tabular}{lc}
\hline $\begin{array}{l}\text { ALTITUD MEDIA DE CADA } \\
\text { CURVA (msnm) }\end{array}$ & AREA INTERVALO DE CURVAS (km) \\
\hline 200 & 37,031 \\
\hline 400 & 85,063 \\
\hline 600 & 199,474 \\
\hline 800 & 290,092 \\
\hline 1000 & 478,160 \\
\hline 1200 & 415,601 \\
\hline 1400 & 228,012 \\
\hline 1600 & 102,130 \\
\hline 1800 & 15,893 \\
\hline 1995 & 5,404 \\
\hline
\end{tabular}

Fuente:Elaboración propia.

círculo de misma área, el Kc es de 1,944, este resultado apunta que la cuenca posee forma oval oblonga a rectangular oblonga.

El índice de homogeneidad presentó un valor 1,79, este resultado define que el cauce principal esta recargado al lado derecho de la cuenca. El índice de asimetría 64,259 indican que la cuenca es fuertemente asimétrica (tabla 4).

La mayor superficie se distribuye entre las cotas 900 a $1.300 \mathrm{msnm}$, y solo $0,09 \%$ de la superficie corresponde a áreas de $1.995 \mathrm{msnm}$ (tabla 5), lo que demuestra una madurez hidrológica. Considerando que esta cuenca se encuentra en la zona de la cordillera de la Costa y que las altitudes de esta cordillera no sobrepasan los 
Tabla 4

Resultados de los Parámetros morfométricos de la cuenca del Salado Bajo.

\begin{tabular}{|c|c|c|}
\hline Variable & Parámetro (unidades; sigla) & Expresión o herramienta de cálculo \\
\hline \multirow{8}{*}{ 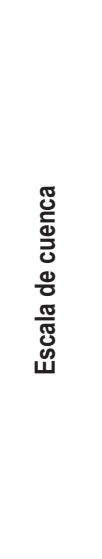 } & Área $\left(\mathrm{km}^{2} ; \mathrm{A}\right)$ & $1.856,686$ \\
\hline & Rectángulo equivalente $(\mathrm{km} ; \mathrm{L})$ & $\begin{array}{l}L_{\text {mayor }}=134,77 \\
I_{\text {menor }}=13,75\end{array}$ \\
\hline & Área de la vertiente mayor (este) $\left(\mathrm{km}^{2}\right)$ & $1.193,102$ \\
\hline & Área de la vertiente menor (oeste) $\left(\mathrm{km}^{2}\right)$ & 663,765 \\
\hline & Perímetro $(\mathrm{km})$ & 297,045 \\
\hline & Longitud (km) & 77,241 \\
\hline & Ancho máximo (km) & 39,422 \\
\hline & Diámetro (km) & 48,623 \\
\hline \multirow{18}{*}{ 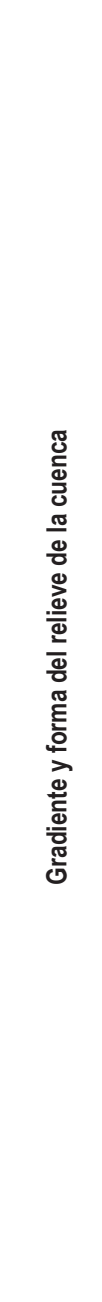 } & Altitud mínima (msnm) & 20 \\
\hline & Altitud máxima (msnm) & 1.995 \\
\hline & Altitud media (msnm) & 1.019 \\
\hline & Pendiente media $(\mathrm{m} / \mathrm{m})$ & 18,59 \\
\hline & Elongación & 0,629 \\
\hline & Perímetro relativo de crenulación & 47,518 \\
\hline & Coeficiente de compacidad (Gravelius) & 1,944 \\
\hline & Índice de alargamiento & 1,959 \\
\hline & Índice de homogeneidad & 1,79 \\
\hline & Índice de asimetría & 64,259 \\
\hline & Factor forma & 0,311 \\
\hline & Alargamiento de Caquot & 1,792 \\
\hline & Radio de elongación & 0,629 \\
\hline & Radio de circularidad & 0,264 \\
\hline & Índice de forma & 1,944 \\
\hline & Índice entre el largo y el área de la cuenca & 0,026 \\
\hline & Coeficiente de forma & 0,323 \\
\hline & Coeficiente de redondez & 2,523 \\
\hline
\end{tabular}

Fuente: elaboración propia 
Tabla 5

Resultados de la relación altimetría/área.

\begin{tabular}{|c|c|c|c|}
\hline Cota altimétrica (msnm) & Área (km2) & Área acumulada (km2) & Porcentaje de área (\%) \\
\hline 118 & 15,154 & 15,154 & 0,81 \\
\hline 217 & 27,497 & 42,651 & 1,48 \\
\hline 316 & 37,193 & 64,69 & 2,00 \\
\hline 415 & 52,031 & 89,224 & 2,80 \\
\hline 513 & 84,54 & 136,571 & 4,55 \\
\hline 612 & 120,152 & 204,692 & 6,47 \\
\hline 711 & 130,728 & 250,88 & 7,04 \\
\hline 810 & 161,887 & 292,615 & 8,71 \\
\hline 908 & 180,633 & 342,52 & 9,72 \\
\hline 1007 & 298,111 & 478,744 & 16,05 \\
\hline 1106 & 244,582 & 542,693 & 13,17 \\
\hline 1205 & 160,174 & 404,756 & 8,62 \\
\hline 1303 & 124,661 & 284,835 & 6,71 \\
\hline 1402 & 97,921 & 222,582 & 5,27 \\
\hline 1501 & 64,308 & 162,229 & 3,46 \\
\hline 1600 & 35,993 & 100,301 & 1,93 \\
\hline 1698 & 11,814 & 47,807 & 0,63 \\
\hline 1797 & 3,994 & 15,808 & 0,21 \\
\hline 1896 & 3,721 & 7,715 & 0,20 \\
\hline 1995 & 1,767 & 5,488 & 0,09 \\
\hline
\end{tabular}

Fuente: elaboración propia

$2.000 \mathrm{msnm}$ en este sector, este resultado indica potencial erosivo y predominante transporte de sedimentos y agua.

El análisis de la curva hipsométrica (figura 3) permitió identificar que se encuentra en un estado intermedio de evolución geomorfológica. La ausencia de convexidad o concavidad expresiva con una curva suave hacia abajo demuestra que el área de la cuenca encajaría en la fase madura definida por Davis (1899) y Baker \&
Twidale (1991). Es una cuenca de sistema montañoso en equilibrio, con abanicos aluviales y valles profundos de piedemonte. Los procesos erosivos son agradativos y degradativos en relativo equilibrio.

El análisis de los resultados del ordenamiento y jerarquización de los canales que conforman la red de drenaje corresponden a un sistema hidrogeomorfológico de orden 7 (figura 3). Esta magnitud es un indicador importante y significativo de la extensión y ramificación 


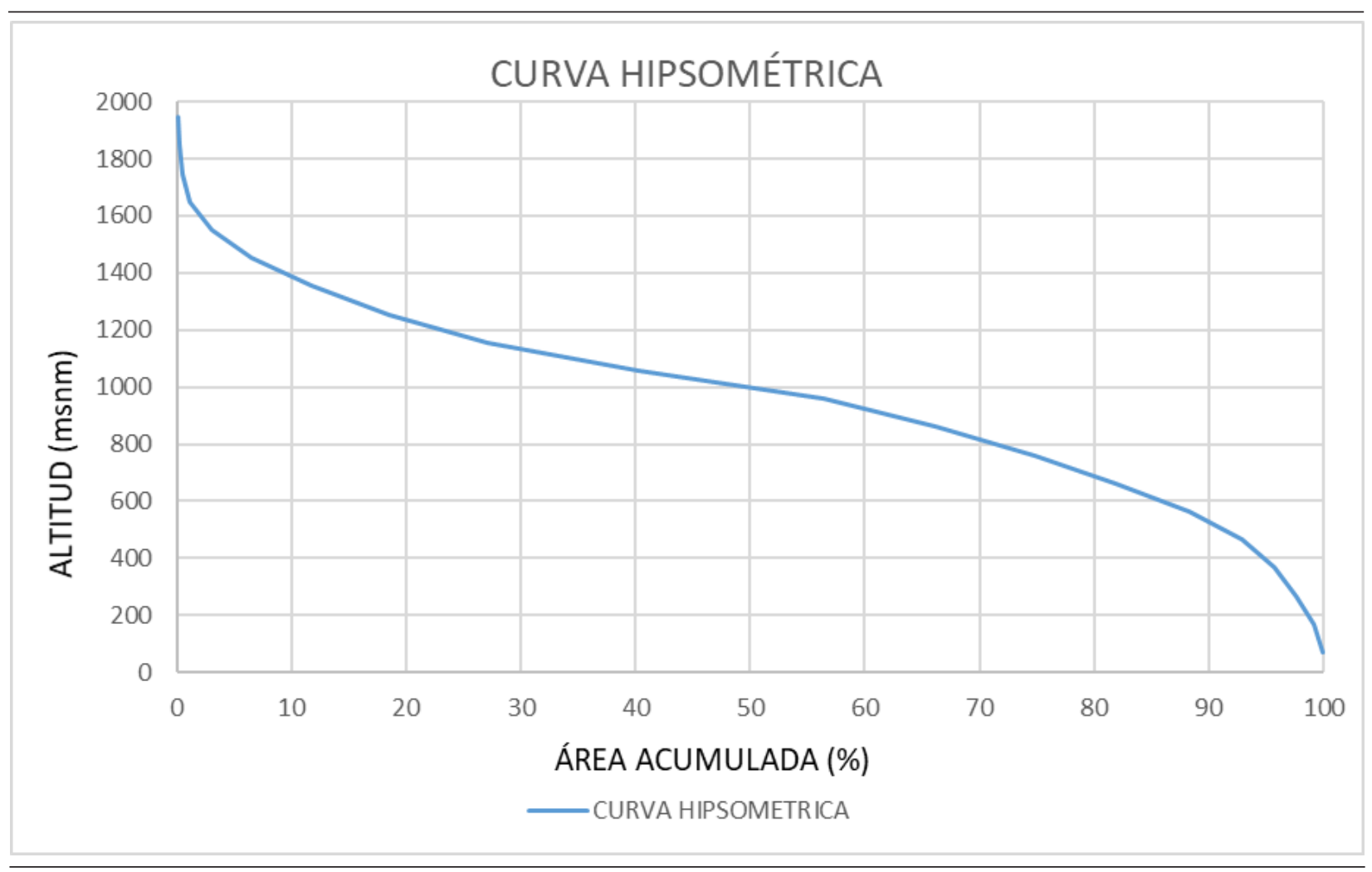

Figura 3. Curva hipsométrica de la cuenca del Salado Bajo. Fuente: elaboración propia.

de la red de drenaje, considerando la pequeña superficie del sistema y su densidad de drenaje media.

Para determinar el tiempo de concentración se utilizó la fórmula de Passini (Ecuación 5), dando por resultado un tiempo de concentración de 2,49 horas, y la velocidad media de flujo se calculó en base a la longitud del cauce principal y el tiempo de concentración anteriormente obtenido, el resultado fue de $0,0315 \mathrm{~h}$.

Los cursos de agua de la red son de cortos recorridos con valores promedios por orden que oscilan entre $10 \mathrm{a}$ $40 \mathrm{~km}$ (corrientes de orden 1, 2, 3, 4, 5 y 6) y 78,71 km (corriente de orden 7). El 64,18\% de las corrientes son de orden 1 con longitud de drenaje promedio de $40 \mathrm{~km}$, lo cual influye significativamente en la reducción de los tiempos de concentración de la cuenca en presencia de tormentas (figura 4).

El canal principal de la red correspondiente a la parte baja del río Salado y la quebrada Saladillo, que en esta zona alcanza su orden 7 aproximadamente a los $919 \mathrm{msnm}$ y posee longitud de $78,71 \mathrm{~km}$ hasta la desembocadura en el mar. Los perfiles topográficos de los tributarios de orden 6 presentaron los siguientes valores: río Salado con
$500 \mathrm{msnm}$ y quebrada de las Ánimas con $1.000 \mathrm{msnm}$ (figura 5). Esto indica que, durante eventos climáticos de precipitación extrema producirían flujos donde se alcanzan caudales máximos importantes desde su curso medio encajado en un cañón montañoso.

La densidad de drenaje fue clasificada en escalas que están relacionadas a la capacidad textural de escorrentía superficial y absorción del suelo. La densidad de drenaje de la cuenca está entre 1,1 a 4,2 km/ $\mathrm{km}^{2}$.

Los valores entre 1,1 a $1,5 \mathrm{~km} / \mathrm{km}^{2}$ presentan textura de drenaje gruesa, donde la escorrentía superficial es menor, la roca es más resistente, aunque más permeable, y los suelos tienen elevada permeabilidad. Este rango obtuvo área $837,18 \mathrm{~km}^{2}$, corresponde a $82 \%$ del área total de la cuenca.

Los valores que varían entre 1,8 a $2,1 \mathrm{~km} / \mathrm{km}^{2}$ son de textura mediana, la escorrentía y la permeabilidad del suelo son intermedios, corresponde a $165,26 \mathrm{~km}^{2}$, esto representa el 16,25\% del área total de la cuenca.

Los valores iguales a $4,2 \mathrm{~km} / \mathrm{km} 2$ indican textura fina, en lo cual la escorrentía superficial es alta, así como la 


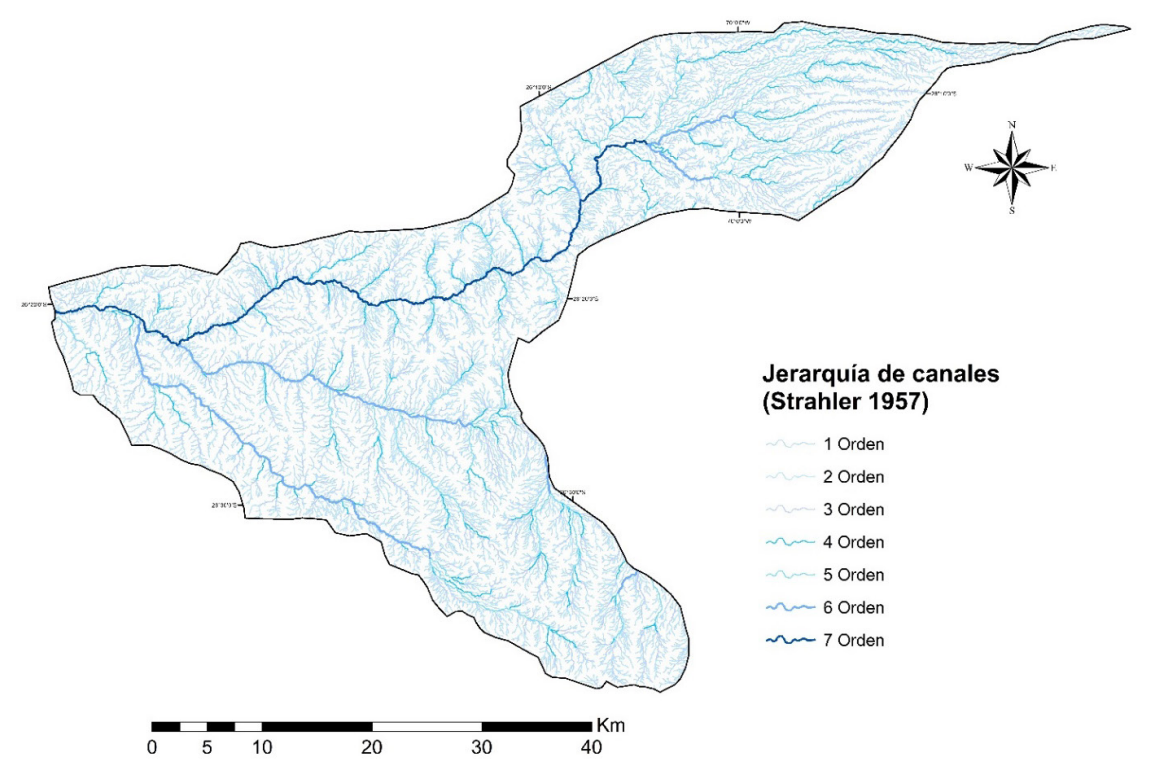

Figura 4. Distribución de los canales según orden jerárquico establecido por la clasificación de Strahler (1957). Fuente: elaboración propia

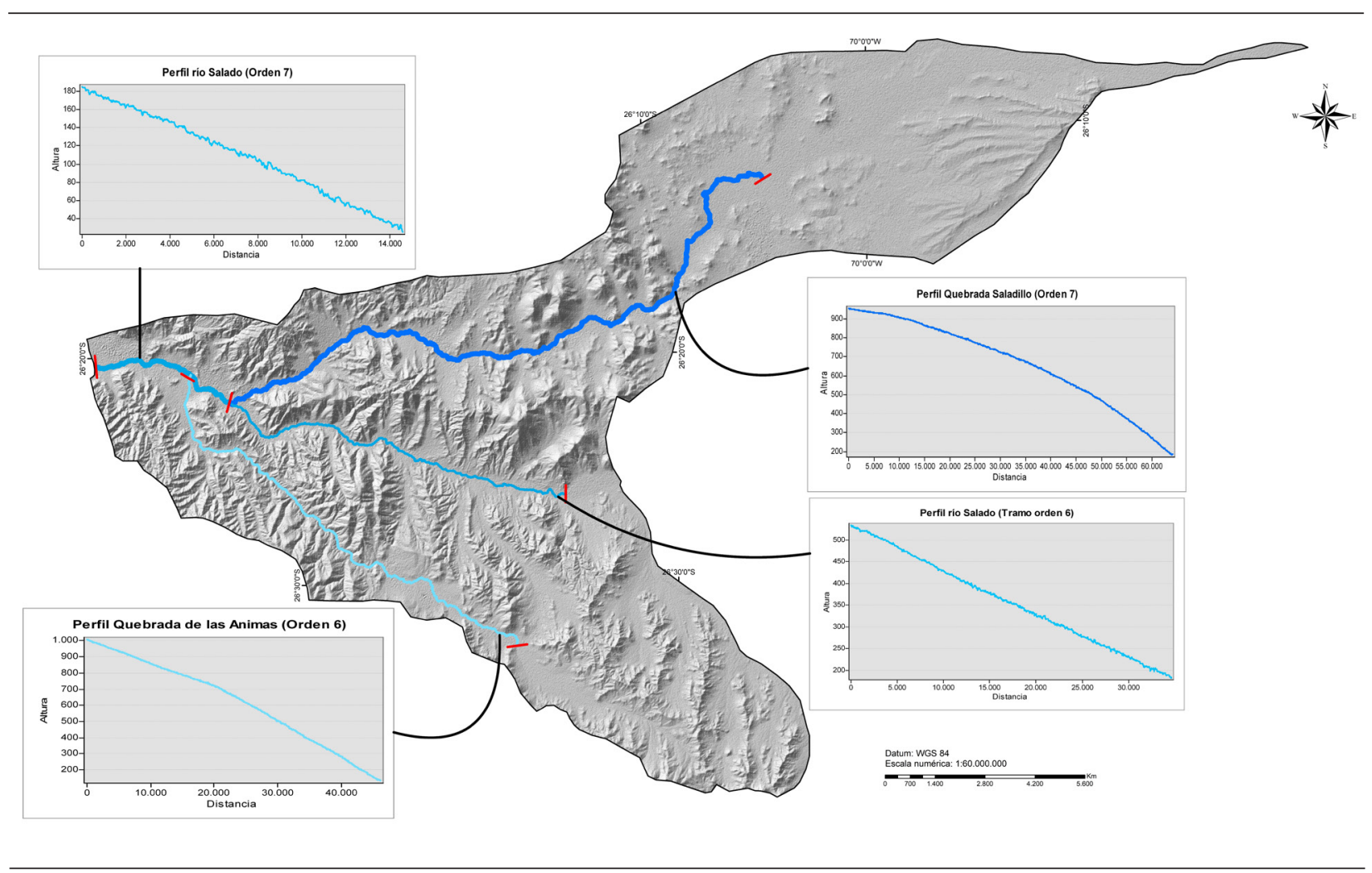

Figura 5. Jerarquía de los canales de orden 6 y 7 , y respectivos perfiles topográficos. Fuente: elaboración propia. 


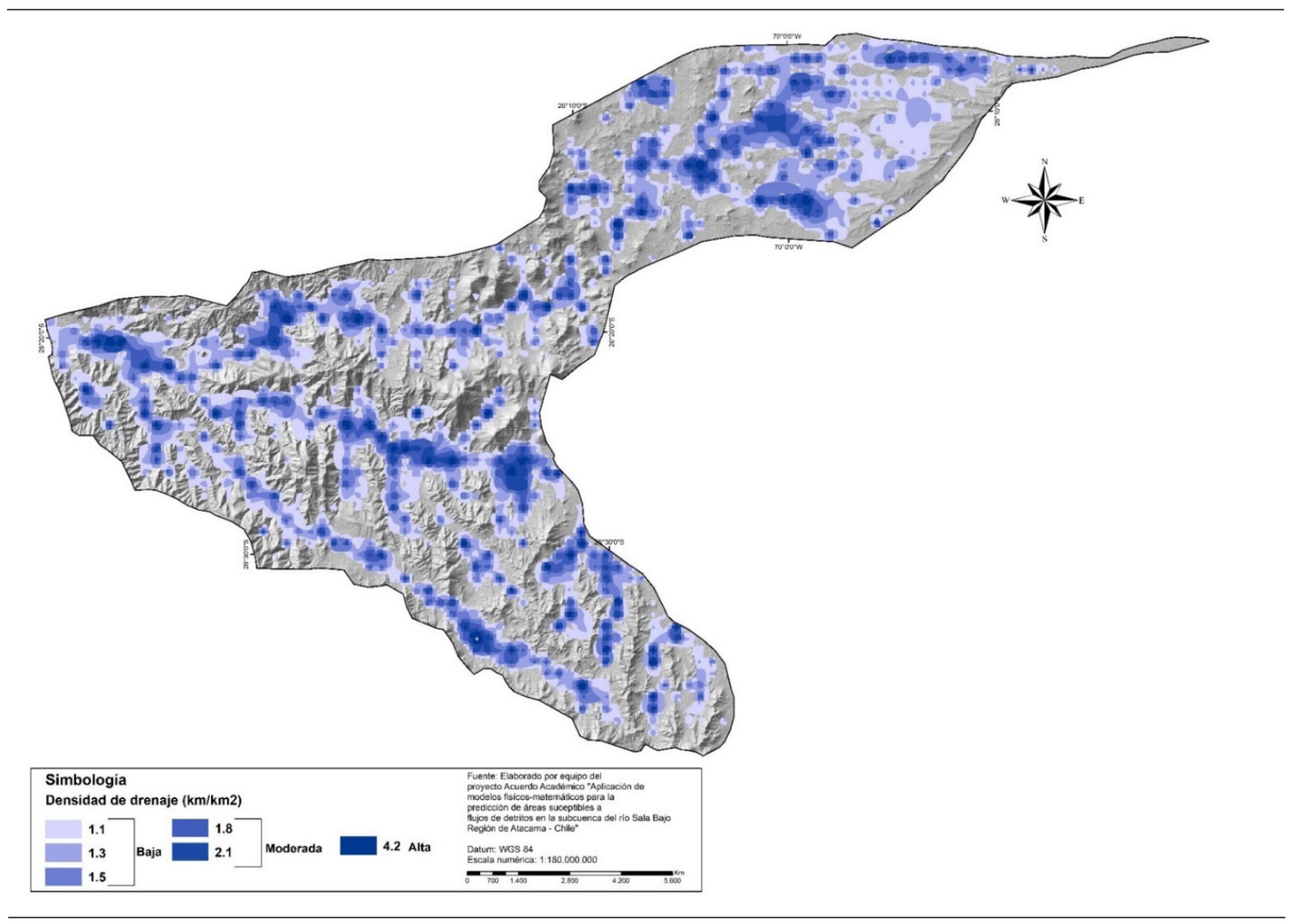

Figura 6. Densidad de la red de drenaje de las cuenca hidrográfica del Salado Bajo. Fuente: elaboración propia

impermeabilidad de suelo, corresponde a $13,98 \mathrm{~km}^{2}$, siendo el 1,3\% del área total de cuenca.

También se calculó la densidad de drenaje del cauce principal (orden 7) y se obtuvo valor de $4,2 \mathrm{~km} / \mathrm{km}^{2}$, siendo un drenaje alto de textura fina (figura 6). De acuerdo con los resultados, es esencial resaltar que las escasas precipitaciones que se registran en la zona, y la vegetación bastante reducida, donde la superficie de la cuenca mayormente es de suelo expuesto, contribuyen con la susceptibilidad del sustrato litológico a los procesos erosivos.

La densidad de drenaje predominante en las áreas entornos de los cauces son las de valores bajos y medianos. En las zonas de densidad mediana y alta la superficie experimenta escorrentía laminar antes de concentrarse en los cauces de la red, por lo que sería un indicador de alta tasa de erodabilidad, principalmente en las áreas de alta densidad.

Considerando que la erodabilidad indica la susceptibilidad de éste a la disgregación, y depende primeramente de las características físicas del suelo. Aun siendo la parte baja del río Salado, y que este recibe sedimentos de las cuencas altas, la cuenca analizada también presenta valores propios de susceptibilidad del suelo, principalmente en los cauces y en las terrazas fluviales.

\section{Discusión}

La caracterización de los parámetros morfométricos es la información básica fundamental para establecer las relaciones entre la hidrología y la geomorfología que determinan la evolución del paisaje. De esta forma, los resultados de los parámetros de escala sirvieron de base para los cálculos de los parámetros de forma y gradiente. Según los resultados obtenidos por tales parámetros, la cuenca posee forma alargada. Además, el Kc indica forma oval oblonga a rectangular oblonga.

Cuencas alargadas presenta respuestas difusas de tormentas de caudales (Matauco, 2004). Esta característica es un referente para determinar la dinámica de la escorrentía superficial, que en este caso es una dinámica fluvial 
rápida. Sistemas fluviales rápidos presentan gran potencial erosivo, lo que resulta en un mayor desarrollo de la energía cinética durante el proceso de arrastre de los sedimentos desde la parte más elevada hasta el nivel de base (Cheng et al., 2005). Además, la forma alargada indica una característica del sistema de drenaje, donde el cauce principal posee mayor longitud que la totalidad de los tributarios. Esta característica es un indicador de la alta susceptibilidad de la cuenca a flujos rápidos.

Conjuntamente, el resultado del índice de compacidad (Kc) corrobora con los resultados anteriores, definiendo que la forma oval oblonga a rectangular oblonga es una característica de una cuenca con un sistema de drenaje sujeto a crecidas de menor magnitud, o de crecidas solamente en el nivel de base de la cuenca.

Los análisis de los resultados de altitudes máxima, mínima y media, en conjunto con la pendiente media de la cuenca indican que el relieve es fuertemente accidentado, y correlacionando este resultado con la geomorfología de quebradas y con los resultados obtenidos del tiempo de concentración y velocidad media del flujo, donde se observan que los canales se encuentran encajados entre valles, se concluye que la morfología interviene directamente en la velocidad del transporte del material erosionado durante precipitaciones.

La curva hipsométrica corroboró en identificar que la cuenca se caracteriza por poseer paisaje con una red de drenaje ampliamente jerárquica, donde sus canales tendrían valles profundos e incrustados, pero al inicio del proceso de aumento de la amplitud lateral de estos valles. En la etapa de madurez, las cimas comenzarían a mostrar una disminución más pronunciada desde el fondo de los valles a medida que la profundización de los valles se redujera más rápidamente en comparación con la fase juvenil. Esta característica también puede estar relacionada con el entorno en el que se encuentra la cuenca en la cordillera de la Costa, un sector más bajo de la cordillera andina con pendientes más suaves. No obstante, la definición de cuenca montañosa de clima árido en equilibrio, el término cuenca en equilibrio no exime la ocurrencia de flujos de sedimentos rápidos, y esta afirmación se refleja en el resultado del tiempo de concentración.

El cauce de orden 7 también es un resultado que indica el potencial de transporte de sedimentos. Además, asociado al resultado del tiempo de concentración, reafirma el potencial erosivo y la susceptibilidad a la ocurrencia de flujos de detritos y barros de alta magnitud, considerado el tipo de hidrología y la forma que posee la cuenca en cuestión.

La red de drenaje está constituida por canales de cortos recorridos y régimen intermitente que definen un patrón de drenaje de tipo subdendrítico. El cual es determinado fundamentalmente por las pendientes de los canales de órdenes 2 y 3 , que producen un cierto grado de paralelismo. La existencia de sistemas de fracturas (diaclasas y fallas) y los desniveles topográficos entre bloques tectónicos, también ejercen control en la disposición y distribución de las corrientes que conforman la red de drenaje.

Los resultados obtenidos en relación con los canales de drenaje corroboran con el modelo de drenaje presentado por Mourier et al. (2008), en el cual los canales de bajo orden casi siempre están saturados por material de deposición de taludes, y material coluvial proveniente de grandes flujos de detritos y barro, que a su vez se relaciona a eventos meteorológicos extremos, mientras que los canales de orden superior están sujetos al mayor predominio de sedimentos aluviales, predominando la escorrentía hortónica, caracterizada por ser una escorrentía que proviene de múltiples direcciones por cuenta de la ausencia de cobertura vegetal típica en las zonas áridas.

\section{Conclusiones}

Fue posible caracterizar cuantitativamente la cuenca del Salado Bajo mediante el uso de la aplicación integrada de parámetros físicos morfométricos.

La forma alargada de la cuenca (jerarquía 7), de geometría rectangular oblonga con presencia de una red de drenaje efectiva de alto potencial erosivo y con parámetros estructuradores de superficies de clima seco, indican que la cuenca del Salado Bajo presenta una susceptibilidad a flujos de detritos y barro.

La cuenca presentó puntos con altos valores densidad de drenaje $\left(4,2 \mathrm{~km} / \mathrm{km}^{2}\right)$, fenómeno hidromorfológico asociado a una potencial ocurrencia de crecidas. Por lo tanto, se recomienda realizar estudios que incluyan la caracterización pedológica, de sustrato rocoso y el régimen de precipitación, variable detonante que demuestra tener un alto impacto en el conjunto de elementos físiconaturales observados.

Se sugiere, para estudios futuros, determinar las áreas específicas de la cuenca que presenten mayor o menor grado de susceptibilidad, y así elaborar una zonificación de aquellas expuestas a tal fenómeno, mediante: a) 
caracterización de componentes físicos complementarios del paisaje, tales como la permeabilidad del suelo y la erosión; y b) aplicación de modelos erosivos y modelos matemáticos de remociones en masa para la zonificación de la susceptibilidad..

\section{Referencias}

Abuzied, S., Yuan, M., Ibrahim, S., Kaiser, M. \& Saleem, T. (2016). Geospatial risk assessment of flash floods in Nuweiba area, Egypt. Journal of Arid Environments, 133, 54-72. https://doi.org/10.1016/j. jaridenv.2016.06.004

Bajirao, T. S., Kumar, A. \& Kumar, P. K. (2019). Application of remote sensing and GIS for morphometric analysis of watershed: A Review. International Journal of Chemical Studies. IJCS, 7(2), 709-713. https://www.researchgate.net/ publication/331981911_Application_of_remote sensing_and_GIS_for_morphometric_analysis_of watershed_A_Review

Baker, V. R. \& Twidale, C. R. (1991). The reenchantment of geomorphology. Geomorphology, 4(2), 73-100. https://doi.org/10.1016/0169-555X(91)90021-2

Bertolini, W. Z., Deodoro, S. C., \& Boettcher, N. (2019). Análisis morfométrico de la cuenca del río Barra Grande-Oeste de Santa Catarina. Revista Brasileira de Geomorfologia, 20(1). http://dx.doi.org/10.20502/ rbg.v20i1.1506

Bhattacharya, R. K., Chatterjee, N. D., Acharya, P., \& Das, K. (2021). Morphometric analysis to characterize the soil erosion susceptibility in the western part of lower Gangetic River basin, India. Arabian Journal of Geosciences, 14(6), 1-22. https://doi.org/10.1007/ s12517-021-06819-8

Bogale, A. (2021). Morphometric analysis of a drainage basin using geographical information system in Gilgel Abay watershed, Lake Tana Basin, upper Blue Nile Basin, Ethiopia. Applied Water Science, 11(7), 1-7. https://doi.org/10.1007/s13201-021-01447-9

Buccolini, M., Coco, L., Cappadonia, C., \& Rotigliano, E. (2012). Relationships between a new slope morphometric index and calanchi erosion in northern Sicily, Italy. Geomorphology, 149-150, 41-48. https:// doi.org/10.1016/j.geomorph.2012.01.012
Cheng, J. D. (2005). Hydrometeorological and landuse attributes of debris flows and debris floods during typhoon Toraji, July 29-30, 2001 in central Taiwan. Journal of Hydrology, 306(1-4), 161-173. https://doi. org/10.1016/j.jhydrol.2004.09.007

Contreras, J. P., Ramírez, C., Garrido, N., \& Núñez, G. (2015). Caracterización hídrica y geológica de los aluviones del 25 y 26 de marzo de 2015 en la cuenca del Río El Salado, Región de Atacama, Chile. En Geología Para El Siglo XXI. Presented at the XIV Congreso Geológico Chileno, La Serena, Chile (pp. 785-788). http://sitiohistorico.sernageomin.cl/pdf/ congresogeo/50.pdf

Cordeiro, T. S., De Abreu, H.A., Da Silva, P.R., Maciel, R., Fernandes, D., Barbosa, D., Cordeiro, J., \& Cordeiro, J. (2019). Morphometric analysis of hydrographic basin of Ribeirão Candidópolis, Itabira (MG). Research, Society and Development, 8(1), e1581529. https://doi.org/10.33448/rsd-v8i1.529

Cornejo, P., Riquelme, R. \& Mpodozis, A. J. (1998). Mapa Geológico de la Hoja Salvador, Región de Atacama, Chile. Servicio Nacional de Geología y Minería (SERNAGEOMIN), Mapas Geológicos (escala 1: 100,000) 7.

Cornejo, P., Mpodozis, C., \& Tomlinson, A.J. (1999). Hoja Salar de Maricunga, Región de Atacama. Servicio Nacional de Geología y Minería (SERNAGEOMIN), Mapas Geológicos (escala 1: 100,000) 7.

Cortés, I., Ríos, J., Gaete, L., \& Tchernitchin, A. (2015). Metales pesados en muestras ambientales de la Región de Atacama. Situación postaluviones. En Abstracts XIV Chilean Geological Conference: Geology for the 21st century, La Serena, Chile (pp. 302-305). https://biblioteca.sernageomin.cl/opac/ DataFiles/14905_v3_pp_302_305.pdf

Da Conceição D. P., Xavier, R. A., da Silva Seabra, V., \& da Silva, R. M. (2020). Análise Morfométrica Da Bacia Do Alto Rio Paraíba, Região Semiárida Do Estado Da Paraiba Morphometric Analyses Of High Paraiba River Basin, Semiárid Region Of Paraiba State. Revista Brasileira de Geomorfologia, 21(3). http://dx.doi.org/10.20502/rbg.v21i3.1757 
Da Silva Alves, K. M. A., de Lira, D. R., D’Avila, M. C. P., de Araújo, M. K., \& de Barros, C. A. C. (2020). Mapeo De Susceptibilidad De Flujo De Detritos Para La Provincia De Chañaral-Chile. Revista Brasileira de Geomorfologia, 21(3). http://dx.doi.org/10.20502/ rbg.v21i3.1743

Davis, W.M. (1899). The geographical cycle. The Geographical Journal, 14(5), 478-504. https://www. jstor.org/stable/1774538? seq=1

De Antueno, L., Gaspari, F. J., \& Guaraca, A. G. (2020). Análisis del efecto del cambio en el uso del suelo sobre el escurrimiento en la cuenca alta del río Sauce Chico, Argentina. Revista Estudios AmbientalesEnvironmental Studies Journal, 8(1), 20-42. https:// doi.org/10.47069/estudios-ambientales.v8i1.659

Fonseca, B. M., \& Augustin, C. H. R. R. (2014). Análise morfométrica de bacias de drenagem e sua relação com a estrutura geológica na serra do Espinhaço Meridional-MG. Revista Brasileira de Geomorfologia, 15(2). http://dx.doi.org/10.20502/rbg.v15i2.296

Gil, V., Volonte, A., \& Campo, A. M. (2019). Índices Morfométricos A Diferentes Escalas Aplicados Al Peligro De Crecidas En Cuencas Pequeñas. Cuenca Del Arroyo San Bernardo, Argentina. Revista Brasileira de Geomorfologia, 20(4). http://dx.doi. org/10.20502/rbg.v20i4.1598

Godínez-Tamay, A., Castillo, M., Ferrari, L., \& OrtegaGutiérrez, F. (2020). Assessing landscape response to tectonics in the Jalisco block and adjacent areas (west-central Mexico) using topographic analysis. Journal of South American Earth Sciences, 98, 102. https://doi.org/10.1016/j.jsames.2019.102469

Godoy, E. \& Lara, L. (1998). Hojas Chañaral y Diego de Almagro, Región de Atacama, escala 1:100.000. SERNAGEOMIN, Mapas Geológicos (5 -6), 1 mapa col. pleg, Santiago.

Godoy, E. \& Lara, L. (2005). Hoja El Salvador Occidental, Región de Atacama. Servicio Nacional de Geología y Minería. Carta Geológica de ChileSerie Geología Básica, 90.

Grijalba Gómez, V. M. (2016). Geología y análisis histórico-meteorológico del aluvión de marzo de 2015 en Chañaral, Atacama. Universidad de Chile. http://repositorio.uchile.cl/handle/2250/140039
Harlin, J. M. (1978). Statistical moments of the hypsometric curve and its density function. Journal of the International Association for Mathematical Geology, 10(1), 59-72. https://doi.org/10.1007/ BF01033300

Harsha, J., Ravikumar, A. S., \& Shivakumar, B. L. (2020). Evaluation of morphometric parameters and hypsometric curve of Arkavathy river basin using RS and GIS techniques. Applied Water Science, 10(3), 1-15. https://doi.org/10.1007/s13201-020-1164-9

Jothimani, M., Dawit, Z., \& Mulualem, W. (2021). Flood Susceptibility Modeling of Megech River Catchment, Lake Tana Basin, North Western Ethiopia, Using Morphometric Analysis. Earth Systems and Environment, 5(2), 353-364. https://doi.org/10.1007/ s41748-020-00173-7

Lin, P., Pan, M., Wood, E. F., Yamazaki, D., \& Allen, G. H. (2021). A new vector-based global river network dataset accounting for variable drainage density. Scientific data, 8(1), 1-9. https://doi.org/10.1038/ s41597-021-00819-9

Lorca, M. (2016). Proyecciones Del Legado MineroIndustrial en La Provincia De Chañaral, Región De Atacama, CHILE1. Diálogo andino, 51, 45-56. http:// dx.doi.org/10.4067/S0719-26812016000300045

Matauco, A. I. G. (2004). Análisis morfométrico de la cuenca y de la red de drenaje del río Zadorra y sus afluentes aplicados a la peligrosidad de crecidas. Boletín de la Asociación de Geógrafos españoles, 38, 311-330. https://dialnet.unirioja.es/servlet/ articulo? codigo $=1079160$

Monroy, O. (2017). Aluviones e inundaciones en la historia de la provincia de Chañaral. Siglos XIX, XX y XXI. Gráfica Pamela Díaz Castro.

Mourier, B., Walter, C., \& Merot, P. (2008). Soil distribution in valleys according to stream order. Catena, 72(3), 395-404. https://doi.org/10.1016/j. catena.2007.07.012

Oliveira, G. P., Tavares, B. D. A. C., \& de Barros Correa, A. C. (2019). Compartimentación geomorfológica del Macizo de Martins-Portalegre-Ne (Brasil). Revista Contexto Geográfico, 3(6), 01-11. https:// doi.org/10.28998/contegeo.v3i6.6805 
Othman, A. A., Gloaguen, R., Andreani, L., \& Rahnama, M. (2018. Improving landslide susceptibility mapping using morphometric features in the Mawat area, Kurdistan Region, NE Iraq: Comparison of different statistical models. Geomorphology, 319, 147-160. https://doi.org/10.1016/j.geomorph.2018.07.018

Pereira, P. S. P. M. L., \& Mendes, L. D. (2019). Morfometría de cuenca hidrográfica urbanizada: Un análisis del río Iguaçu-Sarapuí, Baixada Fluminense $(\mathrm{RJ})$, para la evaluación de parámetros de susceptibilidad a la ocurrencia de crecidas e inundaciones. Revista Continentes, 13, 34-48. https://www.revistacontinentes.com.br/index.php/ continentes/article/view/195/157

Rahmati, O., Samadi, M., Shahabi, H., Azareh, A., RafieiSardooi, E., Alilou, H., \& Shirzadi, A. (2019). SWPT: An automated GIS-based tool for prioritization of sub-watersheds based on morphometric and topohydrological factors. Geoscience Frontiers, 10(6), 2167-2175. https://doi.org/10.1016/j.gsf.2019.03.009

Ramirez, F. J., \& Perez, P. C. (2011). Passing volume calculation system (PVCS): computer software for managing data on watersheds that produce mud flows and the case of Quebrada La Cadena, Antofagasta, Chile. Natural hazards, 59(1), 149-166. https://doi. org/10.1007/s11069-011-9744-5

Shivhare, V., Gupta, C., Mallick, J., \& Singh, C. K. (2021). Geospatial modelling for sub-watershed prioritization in Western Himalayan Basin using morphometric parameters. Natural hazards, 1-17. https://doi.org/10.1007/s11069-021-04957-6

Soares, S. P., Ladeira, F. S. B., \& Liesenberg, V. (2016). Morfometria da bacia do Rio São João, MG: uma proposta de entendimento dos limites e da dinâmica das superfícies erosivas. Revista Brasileira de Geomorfologia, 17(2). http://dx.doi.org/10.20502/ rbg.v17i2.790

Strahler, A. N. (1957). Quantitative analysis of watershed geomorphology. Eos, Transactions American Geophysical Union, 38(6), 913-920. https://doi. org/10.1029/TR038i006p00913

Strahler, A. N., Strahler, A. H. (1989). Geografía Física. Estudios Geográficos, 202, 187.https://search. proquest.com/openview/62c9d54dcf8311786d0fc2d 4a1674c36/1?pq-origsite $=$ gscholar\&cbl $=1818083$
Tomlinson, A., Cornejo, P. \& Mpodozis, C. (1999). Hoja Potrerillos, Región de Atacama. Servicio Nacional de Geología y Minería, SERNAGEOMIN, Mapas Geológicos (escala 1:100.000) 14.

Villegas Cornejo, J. (2017). Re-construyendo la huella de desastre. Los aluviones del río Salado en su paso por Chañaral [Tesis Magister, Universidad Católica de Chile]. Repositorio Institucional. https:// repositorio.uc.cl/handle/11534/27019.

\section{Agradecimientos}

A la Universidad Tecnológica Metropolitana (UTEM) de Chile por el apoyo y financiamiento por medio del Acuerdo Académico para la investigación del proyecto "Modelación de áreas susceptibles a flujos de detritos (aluvión) en la cuenca hidrográfica del río Salado en la región de Atacama de Chile". Se agradece también al Laboratorio de Suelos y Medio Ambiente, dependiente del Departamento de Industria, Facultad de Ingeniería de la UTEM y al Consejo Nacional de Investigación y Técnicas (CONICET - ARGENTINA) por medio de la beca postdoctoral Latinoamérica 2020, por el apoyo en la ejecución del estudio. 